\title{
Highlights
}

\section{Tree Species Classification Using Structural Features Derived From}

\section{Terrestrial Laser Scanning}

Louise Terryn, Kim Calders, Mathias Disney, Niall Origo, Yadvinder Malhi,

Glenn Newnham, Pasi Raumonen, Markku Åkerblom, Hans Verbeeck

- We classify five tree species using structural traits from terrestrial laser scanning

- Size dependency and structural trait convergence complicate species classification

- Defining a targeted application is important to achieve a good classification 


\title{
Tree Species Classification Using Structural Features Derived From Terrestrial Laser Scanning
}

\author{
Louise Terryn ${ }^{\mathrm{a}, *}$, Kim Calders ${ }^{\mathrm{a}}$, Mathias Disney ${ }^{\mathrm{b}, \mathrm{c}}$, Niall Origo ${ }^{\mathrm{d}, \mathrm{b}}$, \\ Yadvinder Malhi ${ }^{\mathrm{e}}$, Glenn Newnham ${ }^{\mathrm{f}}$, Pasi Raumonen ${ }^{\mathrm{g}}$, Markku Akerblom ${ }^{\mathrm{g}}$, \\ Hans Verbeeck ${ }^{\mathrm{a}}$ \\ ${ }^{a}$ CAVElab - Computational \& Applied Vegetation Ecology, Ghent University, Belgium \\ ${ }^{b} U C L$ Department of Geography, Gower Street, London WC1E 6BT, UK \\ ${ }^{c}$ NERC National Centre for Earth Observation (NCEO), UCL, Gower Street, London \\ WC1E $6 B T, U K$ \\ ${ }^{d}$ Earth Observation, Climate and Optical Group, National Physical Laboratory, \\ Teddington, TW11 OLW, UK \\ ${ }^{e}$ Environmental Change Institute, School of Geography and the Environment, University \\ of Oxford, Oxford OX1 3QY, UK \\ ${ }^{f}$ Land and Water, Commonwealth Scientific and Industrial Research Organisation, Black \\ Mountain, ACT, Australia \\ ${ }^{g}$ Mathematics, Tampere University, Tampere, Finland
}

\begin{abstract}
Fast and automated collection of forest data, such as species composition information, is required to support climate mitigation actions. Recently, there have been significant advances in the use of terrestrial laser scanning (TLS) instruments, which facilitate the capture of detailed forest structure. However, for tree species recognition the structural information from TLS has mainly been used to complement spectral information. TLS-only classification studies have been limited in size and diversity of plot forest types. In this paper, we investigate the potential of TLS for tree species classifica-
\end{abstract}

\footnotetext{
*Corresponding author. Louise.Terryn@UGent.be; terrynlouise@outlook.com
} 
tion. We used quantitative structure models to determine 17 structural tree features. These features were computed for 758 trees of five tree species, including two understory species, of a 1.4 hectare mixed deciduous forest plot. Three classification methods were compared: k-nearest neighbours, multinomial logistic regression and support vector machine. We assessed the potential underlying causes for structural differences with principal component analysis. We obtained classification success rates of approximately $80 \%$, however, with producer accuracies for three of the five species ranging from 0 to $60 \%$. Low producer accuracies were the result of a high intra- and low inter-species variability. These effects were, respectively, caused by a high size-dependency of the structural features and a convergence of structural traits across species as a result of the individual tree position in the forest canopy and shade tolerance. Nevertheless, the producer accuracies could be improved through sensitivity vs. specificity trade-offs, with over $50 \%$ for all species being obtainable. The high intra -and low inter-species variability complicate the classification. Furthermore, the classification performance and best classification method greatly depend on its targeted application. In conclusion, this study proves the added value of TLS for tree species classification but also shows that TLS opens up potential for testing and further development of ecological theory.

Keywords: Quantitative structure model, Structural tree features, Terrestrial laser scanning, Tree species classification, Machine learning classifiers 


\section{Introduction}

Forests play a significant role in the mitigation of climate change through their ability to sequester $\mathrm{CO}_{2}$ (Bonan, 2008). Although forest ecosystems are resilient, and many species and ecosystems have adapted historically to changing conditions, future changes are likely to occur at rates that are beyond the natural adaptive capacity of the majority of forest species or ecosystems (Keenan, 2015). The development of methodologies for monitoring and assessing forests impacted by hazardous natural processes is therefore increasingly important, resulting in a demand for fast and automated collection of forest data (Deshayes et al., 2006).

Tree species information is important for accurate biomass estimates, biodiversity studies, gas exchange or forest floor research (Fender et al., 2013; Vesterdal et al., 2008; Hobbie et al., 2006). Information on species composition is traditionally summarised in forest inventories. These inventories are compiled for the effective management of forested ecosystems, as tree species information significantly benefits economic, ecological and technical interests (Gong et al., 1997).

Light detection and ranging (LiDAR) instruments collect 3D point clouds by emitting laser pulses and recording the time it takes for the pulse to return. From the range and the angle of the laser pulses the $(\mathrm{x}, \mathrm{y}, \mathrm{z})$ coordinates are

determined. Analysis of this data can provide information on the organisa- 
tion and structure of trees. Most LiDAR-based species classification studies use airborne laser scanning, but since the early 2000s, there has been a growing interest in terrestrial laser scanning (TLS) as a tool for individual tree and forest plot measurements (Newnham et al., 2015). However, tree species classification from TLS data has only featured in a few studies (Puttonen et al., 2010). For instance, Othmani et al. (2013) focused on the 3D geometric texture of bark to classify 75 trees of five tree species (hornbeam, oak, spruce, beech, pine) using the random forest algorithm. They were able to obtain an average overall classification accuracy of $85 \% \pm 5 \%$. More recently, Lin and Herold (2016) classified 40 trees (comprised of four species) based on explicit tree structure parameters using the support vector machine algorithm, obtaining a maximum total accuracy of $90.0 \%$ and a robust total accuracy of $77.5 \%$. A study with a larger sample of 1200 trees and a more automatic solution was presented by Åkerblom et al. (2017). They applied quantitative structure models (QSMs) to obtain structural features of the tree and used these for tree species classification. QSMs are cylinder models which are solely reconstructed using the cartesian $(\mathrm{x}, \mathrm{y}, \mathrm{z})$ coordinates of the 3D point cloud data. QSMs can approximate the branching structure, geometry, and volume of the trees and store geometric and topological properties from which features can be derived (Raumonen et al., 2013; Calders et al., 2015a).

The results of Åkerblom et al. (2017) show that by using single species 
forest plots for training and testing, it is possible to achieve an average classification accuracy above $93 \%$. However, for their preliminary mixed-species forest plot testing, accuracy was lower as neither the classification method parameters or the feature combination were optimised.

We build on the work of Åkerblom et al. (2017) to further test and explore the automated tree species recognition algorithm for mixed species stands including tall as well as understory tree species. We aim to test a classification based only on TLS data in a mixed forest discriminating the five main tree species present: Acer pseudoplatanus, Fraxinus excelsior, Crataegus monogyna, Corylus avellana, Quercus robur. We use structural information, in the form of structural features, from 760 trees to classify these tree species. We do this by first computing and examining the structural tree features for classification. Secondly, we explore the results of a principal component analysis (PCA) on these features to gain insight into the key features that can be used to discriminate the five main tree species. Thirdly, we apply, optimise and compare k-nearest neighbours (KNN)-, multinomial logistic regression (MLR)- and support vector machine (SVM)-classifiers and their parameters for tree species classification.

\section{Study area and data collection}

Wytham Woods (Oxford, UK) is a 404 hectare, ancient semi-natural woodland and one of the most researched areas of woodland in the world 
(Fig. 1). The site has been owned and maintained by Oxford University since 1942. It is a very managed wood where the older, large trees tend to have undergone coppicing and there is some intervention in the understory as well. The mean annual temperature in Wytham Woods is $9.9^{\circ} \mathrm{C}$, and the mean annual rainfall is $744 \mathrm{~mm}$ (The ECN Data Centre, 2019; Butt et al., 2009).

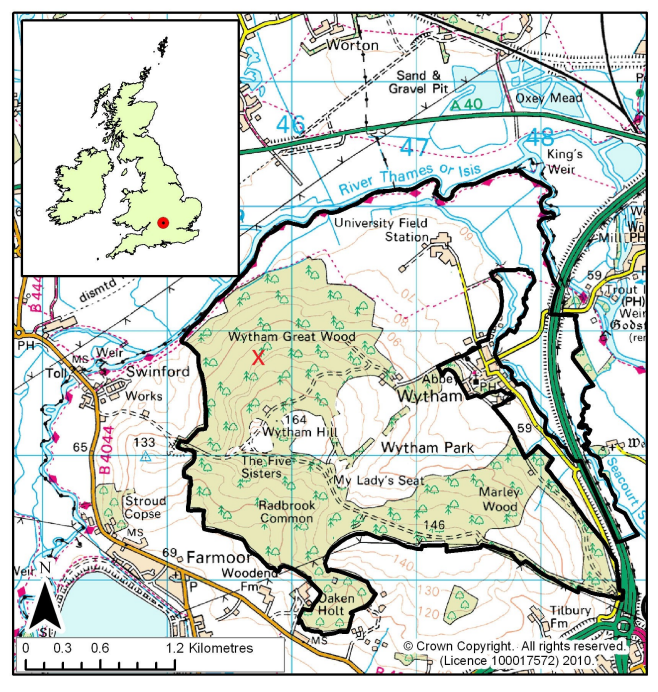

Figure 1: Location and map of Wytham Woods with plot indicated by ' $\mathrm{X}$ ' (The ECN Data Centre, 2019).

The study area is a 1.4 ha plot in Wytham Woods and is part of an 18-hectare long-term Smithsonian forest inventory plot run by Oxford University (Forest GEO Global Earth Observatory Network, 2019). The local origin coordinate $(0,0)$ was measured with differential GPS and located at $51^{\circ} 46^{\prime} 30.2088^{\prime \prime} \mathrm{N}$ and $1^{\circ} 20^{\prime} 20.5692^{\prime \prime} \mathrm{W}$. The 1.4 ha plot has SW-coordinate $(0,100)$ and NE-coordinate $(140,200)$. 
The forest consists of about 23 species but is dominated (approximately 96\%) by five tree species. More specifically, Acer Pseudoplatanus (ACERPS, Sycamore), Fraxinus excelsior (FRAXEX, European/Common ash) and Crataegus monogyna (CRATMO, Common hawthorn) constitute $88 \%$ of the trees. Another $8 \%$ is Corylus avellana (CORYAV, Common Hazel) and Quercus robur (QUERRO, Pedunculate/English oak). Example point clouds of the observed tree species can be found in Fig. 2. As some of the trees are not assigned species or are dead, the dataset consists of 760 identified and living trees of which 550, 84, 66, 35 and 25 trees are ACERPS, FRAXEX, CORYAV, QUERRO and CRATMO trees respectively. Additional information on these five tree species in the 1.4 hectare Wytham Woods plot can be found in Table 1. For all species (excluding QUERRO) young and mature trees are included in the dataset. The stem density in this 1.4 ha study area is 563 stems per hectare. The distribution of the stems of the different species and their DBH is represented in Fig. 3.

\begin{tabular}{lllllll}
\hline & Code & $\#$ & Median DBH $[\mathrm{cm}]$ & DBH range $[\mathrm{cm}]$ & Median height $[\mathrm{m}]$ & Height range $[\mathrm{m}]$ \\
\hline Acer pseudoplatanus & ACERPS & $570(20)$ & 18.60 & $0.50-146.00$ & 16.20 & $1.67-30.50$ \\
Fraxinus excelsior & FRAXEX & $85(1)$ & 18.40 & $3.93-118.00$ & 17.40 & $2.15-28.60$ \\
Corylus avellana & CORYAV & $68(2)$ & 8.33 & $0.50-16.00$ & 7.92 & $0.89-14.00$ \\
Crataegus monogyna & CRATMO & $28(3)$ & 9.83 & $4.09-24.50$ & 8.22 & $1.48-11.30$ \\
Quercus robur & QUERRO & $37(2)$ & 67.90 & $36.60-110.00$ & 19.90 & $10.00-24.40$ \\
\hline
\end{tabular}

Table 1: Overview of the five main tree species in the 1.4 hectare Wytham Woods plot. It includes the number of individuals of each tree species in the dataset, where the amount between brackets is the amount of dead standing trees of that species. Also the mean $\mathrm{DBH}[\mathrm{cm}]$ and $\mathrm{DBH}$ range $[\mathrm{cm}]$ and the mean height $[\mathrm{m}]$ and height range $[\mathrm{m}]$ are given for every tree species.

TLS data were collected by Calders et al. (2018) in leaf-off conditions 

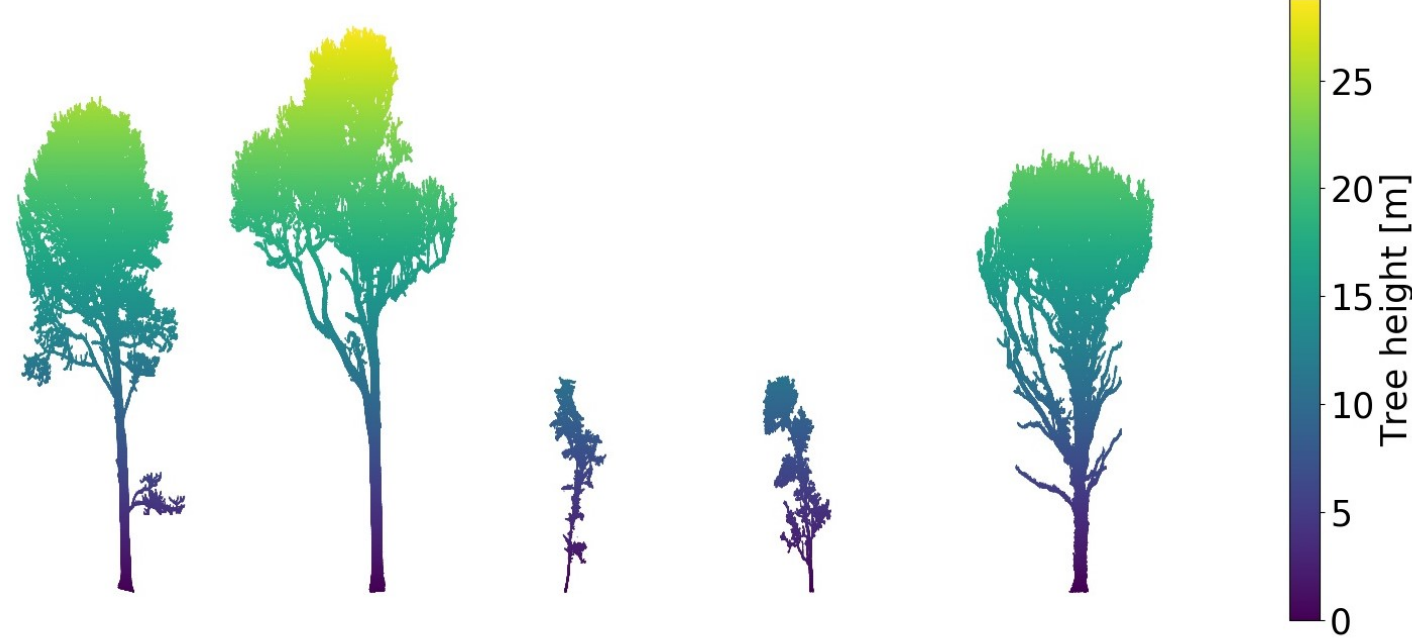

Figure 2: Point clouds of the five main tree species in Wytham Woods coloured by height. From left to right: ACERPS, FRAXEX, CORYAV, CRATMO, QUERRO.

with a RIEGL VZ-400 terrestrial laser scanner (RIEGL Laser Measurement Systems $\mathrm{GmbH}$ ). The instrument has a wavelength of $1550 \mathrm{~nm}$ and a nominal beam divergence of $0.35 \mathrm{mrad}$. The angular sampling for both zenith and azimuth angle is $0.04^{\circ}$ and the azimuth and zenith range are $0^{\circ}-360^{\circ}$ and $0^{\circ}-130^{\circ}$ respectively. The plot was scanned in a $20 \times 20 \mathrm{~m}$ regular grid. Individual trees were extracted with the semi-automatic approach Treeseg (Burt et al., 2019). Full details on the tree segmentation can be found in Calders et al. (2015b). The QSMs were reconstructed from the co-registered TLS data with an approach building on the TreeQSM method by Raumonen et al. (2013) and Calders et al. (2015b), which fits cylinders to the point cloud data following the branch structure of each individual tree (Raumonen, 2019). 


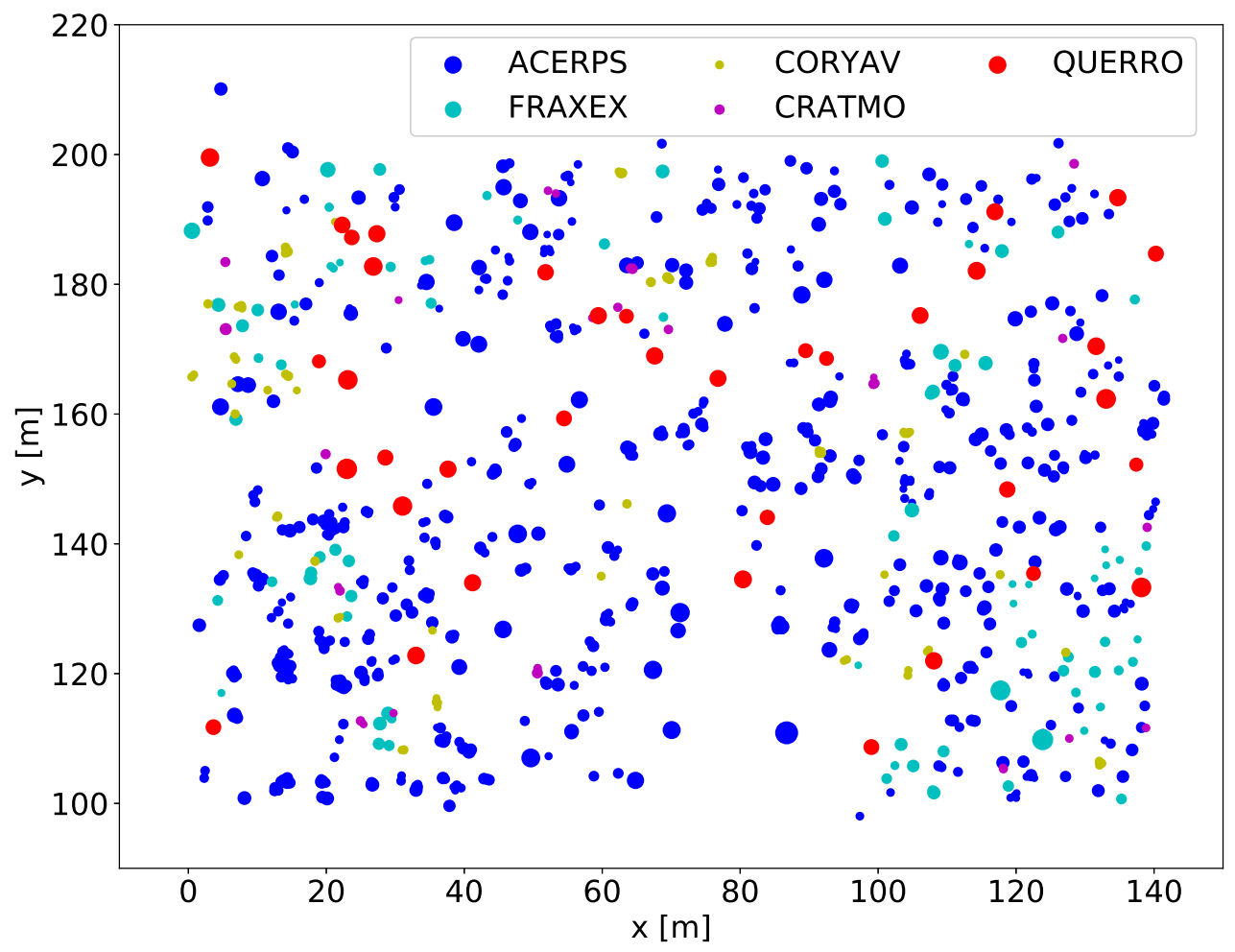

Figure 3: Stem distribution of all the trees in the 1.4 ha study area. The colours dark blue, turquoise, yellow, purple and red represent the tree species ACERPS, FRAXEX, CORYAV, CRATMO and QUERRO respectively. The size of the circles is relative to the $\mathrm{DBH}$ of each tree.

\section{Methods}

\subsection{Feature extraction}

QSMs contain substantial geometric and topological data including branching structure. This is illustrated by the point cloud of a single tree and its QSM in Fig. 4. We extracted 17 structural features from the QSMs of 760 trees. Fifteen structural features were based on those defined by Åkerblom 
et al. (2017) and two additional features were added.

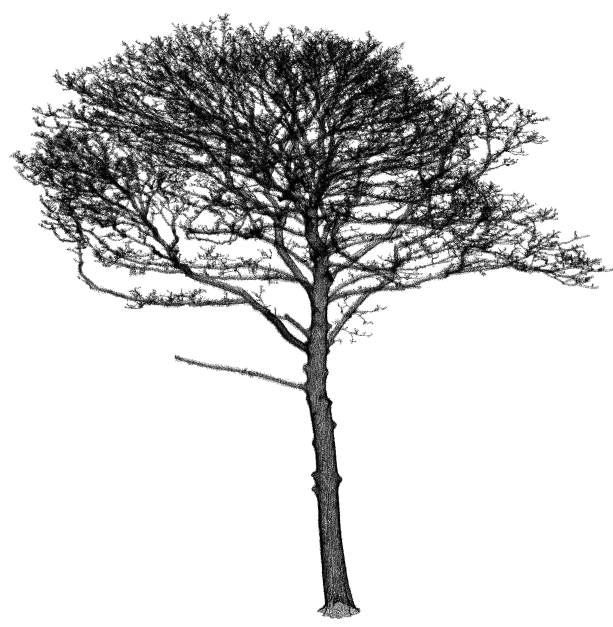

(a) Point cloud

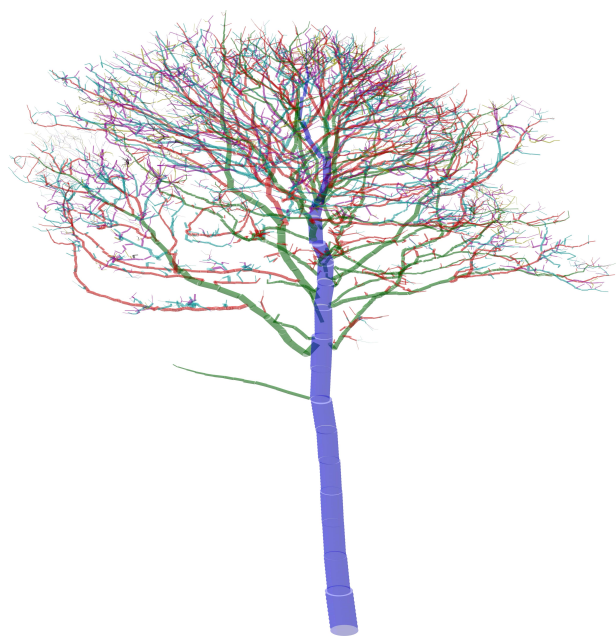

(b) QSM

Figure 4: Illustration of the point cloud and the QSM of an ACERPS tree. The QSM figure is coloured by branching order.

Because the dataset consists of a wide range of stem diameters for four of the five species, we investigated normalisation of features as a function of the DBH. Using DBH-normalised features effectively integrates the DBHdistribution of the trees. Therefore, three out of nine features that show size-dependency were altered. For the other six size dependent features no improved linear normalisation was found. The list of extracted tree features with their description and how their normalisation was adapted can be found in Table 2.

\subsection{Principal Component Analysis}

To obtain an integrated view on the features, we performed a PCA which uses an orthogonal transformation to convert the set of structural features 


\begin{tabular}{|c|c|c|c|}
\hline Number & Name & Description & Remarks \\
\hline 1 & Stem branch angle & $\begin{array}{l}\text { Median of the branching angles of the } 1 \text { st order } \\
\text { branches in degrees. } 0 \text { is upwards and } 180 \text { down- } \\
\text { wards (parallel with the trunk). }\left[^{\circ}\right]\end{array}$ & - \\
\hline 2 & Stem branch cluster size & $\begin{array}{l}\text { Average number of } 1 \text { st order branches inside a } 40 \\
\mathrm{~cm} \text { height interval for } 1 \text { st order branches. Each } \\
\text { branch can only belong to one interval. }\end{array}$ & - \\
\hline 3 & Stem branch radius & $\begin{array}{l}\text { Mean ratio between the } 10 \text { largest } 1 \text { st order } \\
\text { branches measured at the base and the stem ra- } \\
\text { dius at respective height. }\end{array}$ & $\begin{array}{l}\text { Adaptation: mean ratio between the } 10 \text { largest } \\
1 \text { st order branches measured at the base nor- } \\
\text { malised by the tree height. }\end{array}$ \\
\hline 4 & Stem branch length & $\begin{array}{l}\text { Average length of 1st order branches normalised } \\
\text { by DBH. }\end{array}$ & $\begin{array}{l}\text { Adaptation: average length of 1st order branches } \\
\text { normalised by the tree height. }\end{array}$ \\
\hline 5 & Stem branch distance & $\begin{array}{l}\text { Average distance between } 1 \text { st order branches com- } \\
\text { puted using a moving average with a window width } \\
1 \mathrm{~m} \text {. If window is empty average distance in win- } \\
\text { dow is set as half of window width. Normalised by } \\
\text { the DBH. }\end{array}$ & $\begin{array}{l}\text { Adaptation: not normalised and no longer unit- } \\
\text { less }[\mathrm{m}] \text {. }\end{array}$ \\
\hline 6 & Crown start height & $\begin{array}{l}\text { Height of first stem branch in tree crown relative } \\
\text { to tree height. }\end{array}$ & - \\
\hline 7 & Crown height & $\begin{array}{l}\text { Vertical distance between the highest and lowest } \\
\text { crown cylinder relative to tree height. }\end{array}$ & - \\
\hline 8 & Crown evenness & $\begin{array}{l}\text { Crown cylinders divided into } 8 \text { angular bins. Ra- } \\
\text { tio between minimum heights of the highest and } \\
\text { lowest bin. }\end{array}$ & $\begin{array}{l}\text { When one of the bins is empty, the value is set to } \\
\text { zero. }\end{array}$ \\
\hline 9 & Crown diameter / height & Ratio between crown diameter and crown height. & - \\
\hline 10 & DBH / height & Ratio between DBH and total tree height. & - \\
\hline 11 & $\mathrm{DBH} /$ tree volume & Ratio between $\mathrm{DBH}$ and total tree volume. $\left[\mathrm{m}^{-2}\right]$ & - \\
\hline 12 & DBH / minimum tree radius & $\begin{array}{l}\text { Ratio between DBH and the minimum of the ver- } \\
\text { tical bin radius estimates. }\end{array}$ & $\begin{array}{l}\text { The minimum vertical bin diameter is used in- } \\
\text { stead of the radius based on the supplementary } \\
\text { video of Akerblom et al. (2017). }\end{array}$ \\
\hline 13 & Volume below $55 \%$ of the tree & Relative cylinder volume below $55 \%$ of tree height. & $\begin{array}{l}\text { The volume is the branch volume (trunk not in- } \\
\text { cluded). }\end{array}$ \\
\hline 14 & Cylinder length / tree volume & $\begin{array}{l}\text { Ratio between total length of all cylinders and to- } \\
\text { tal tree volume. }\left[\mathrm{m}^{-2}\right]\end{array}$ & $\begin{array}{l}\text { The cylinder length is the branch cylinder } \\
\text { length and the tree volume is the volume of the } \\
\text { branches (trunk not included). }\end{array}$ \\
\hline 15 & Shedding ratio & $\begin{array}{l}\text { The number of branches without children divided } \\
\text { by the number of all branches in the bottom third. }\end{array}$ & $\begin{array}{l}\text { The branches are the stem branches instead of } \\
\text { all branches. Bottom third means the lower third } \\
\text { when the tree is divided in three parts based on } \\
\text { the tree height. }\end{array}$ \\
\hline 16 & Branch angle ratio & $\begin{array}{l}\text { Ratio of the medians of the branching angles of the } \\
\text { 1st order branches and 2nd order branches. }\end{array}$ & New feature. \\
\hline 17 & Relative volume ratio & $\begin{array}{l}\text { Ratio of the percentage volume within } 80 \text { to } 90 \% \text { of } \\
\text { the tree height and the percentage volume within } \\
0 \text { to } 10 \% \text { of the tree height. }\end{array}$ & New feature. \\
\hline
\end{tabular}

Table 2: List of the structural tree features, initiated on the description of Åkerblom et al. (2017). In the last column remarks concerning the description and the feature extraction are added. Adaptation is the adapted form of the feature after feature evaluation to remove size-dependency. 
into a set of linearly uncorrelated variables called principal components. All the features were standardised onto the unit scale using the StandardScaler from the scikit-learn library. The first two most significant (eigenvalue $>1$ ) principal component axes were retained. The third component resulted in a very similar division of the species as principal component 1 but with a larger overlap between the species. Bivariate relationships between traits and principal components were assessed with Spearman's correlation coefficient $r$. High correlation coefficients give insight into what the principal components represent in terms of structural traits.

\subsection{Classification}

We performed the classification, using 17 structural features and three different classifiers, on 758 trees of the five main tree species in Wytham Woods. Two trees were excluded from the dataset before classification as not all of their structural features could be calculated. The QSMs of these trees had no more than one branch and these trees were most likely dead. Feature evaluation revealed that for about half of the features, young ACERPS and FRAXEX trees showed different structural feature distributions compared to the mature trees of both species. Because of this high size-dependency two scenarios were applied. The first scenario considers five classes representing the five main species. In order to improve classification in the second scenario ACERPS and FRAXEX are split into mature and young trees, resulting in 7 classes. This separation was based on a DBH-threshold of $0.2 \mathrm{~m}$ which is 
the point where the height stops increasing linearly with the DBH for both tree species (Butt et al., 2009).

Before the classification we standardised the data so that all features are given a mean of zero and a standard deviation of one. Nested cross validation (Fig. 5) was applied to determine the accuracy of each classifier using fully independent data (Varma and Simon, 2006). We implemented this process with five splits in the outer-validation loop and four splits in the inner-loop. In each outer-loop we used the best parameters of the inner-loops to test the accuracy. Eventually, we computed mean overall test accuracy scores, the number of correctly classified trees divided by the total number of trees, over the five outer-loops for every classifier.

The classification accuracy is not always the most appropriate metric to evaluate the performance of a classifier, especially in the case of imbalanced datasets or when you want to avoid false negatives at the cost of false positives (Story and Congalton, 1986; Pozzolo et al., 2015). Therefore, we also combined the prediction results to obtain aggregated confusion matrices which give an overview of the error types we have made during classification (Story and Congalton, 1986). From the confusion matrices the producer accuracies, which are the number of correctly classified trees of a species divided by the total number of trees of that species, were calculated. 


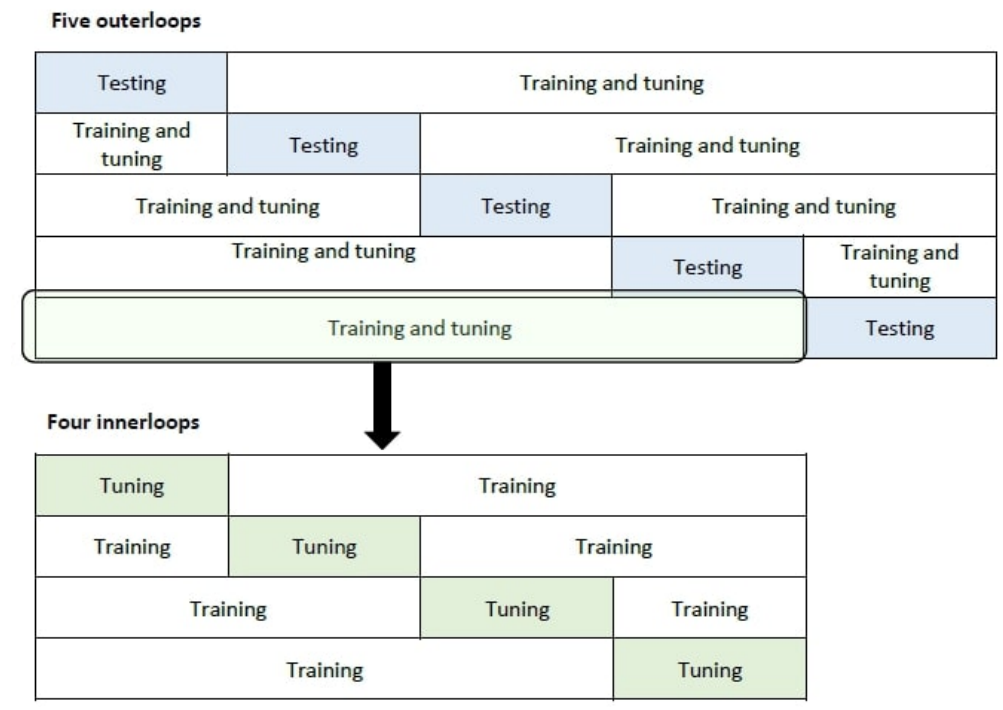

Figure 5: Illustration of nested cross-validation with five outer -and four innerloops. The dataset is split up five times (outerloop) in $20 \%$ testing data and $80 \%$ training and tuning data which is subsequently split up four times (innerloop) in 25\% tuning data and $75 \%$ training data. Optimal parameters are obtained for every innerloop and the best parameter values are chosen for the outerloop. The mean test score is determined based on the test scores for every outerloop.

In the case of imbalanced datasets the concepts of specificity and sensitivity are also better suited than the classification accuracy (Newby et al., 2013). The sensitivity is defined as the fraction of the smallest group that is correctly identified while the specificity is the fraction of the largest group that is correctly classified. Suppose, we have two species groups, ACERPS (largest group) of which we have 548 trees and OTHER of which we have 210 trees. Of the OTHER class the classifier misses 130 predictions (62\%) while we still have a classification accuracy of $80 \%$ because 527 of the 548 ACERPS are correctly classified. So typically while the classifier achieves a high overall classification accuracy and good specificity, the sensitivity is 
poor. We computed these metrics based on the aggregated confusion matrices assuming two classes ACERPS and OTHER (FRAXEX + CORYAV + CRATMO + QUERRO)

The classification was performed for all feature combinations. The combination with the best accuracy score was selected. For specific applications the feature combination could be chosen based on a different performance metric.

\subsubsection{Classifiers}

As the performance of a classifier often depends on the dataset, we applied three different classifiers (Duriqi et al., 2016). The first classification method is the k-nearest neighbour (KNN) classifier where the object is assigned to the class most common among its $\mathrm{k}$ nearest neighbours (Liao and Vemuri, 2002). A second popular classifier is the multinomial logistic re-

gression (MLR) classifier which assigns an observation to the class with the highest probability (James et al., 2013). Thirdly, we implemented support vector machines with the linear, polynomial and radial basis function kernels. All classifiers were implemented in Python (Van Rossum and Drake Jr, 1995) using the scikit-learn library (Pedregosa et al., 2011). Table 3 summarises which parameters were optimised and the range over which they were optimised. 


\begin{tabular}{llll}
\hline Classifier & Parameters & Parameter description & Parameter range \\
\hline KNN & n-neighbours & number of neighbours & 1 to 20 in steps of 1 \\
& metric & distance metric & euclidian, manhattan or chebyshev \\
MLR & $\mathrm{C}$ & inverse of regularisation strength & 0.001 to 100 in logarithmic steps \\
& solver & algorithm used in the optimisation problem & lbfgs, sag or newton-cg \\
SVM & $\mathrm{C}$ & penalty parameter & 0.001 to 100 in logarithmic steps \\
& gamma & kernel coefficient & 0.001 to 100 in logarithmic steps \\
\hline
\end{tabular}

Table 3: Summary of parameter optimisation of the different classifiers. It includes the optimised parameters, their description and the range or option over which they were optimised. The KNN-, MLR- and SVM-classifiers were implemented using sklearn.neighbors.KNeighborsClassifier, sklearn.linear_model. LogisticRegression and sklearn.svm.SVC from the scikit-learn library respectively.

In view of the unbalanced nature of the dataset, the parameter class_weight, optional for the MLR- and SVM-classifiers, was also studied. The default option 'none' means that all classes have a weight equal to one while the 'balanced' option automatically adjusts weights inversely proportional to the class frequencies. We applied both modes for each classifier in each scenario.

\section{Results}

\subsection{Feature evaluation}

Univariate test showed that none of the individual features were significantly different for all observed tree species (Fig. 6). Moreover, a substantial amount of the structural features showed size-dependency despite the normalisation procedure introduced. 

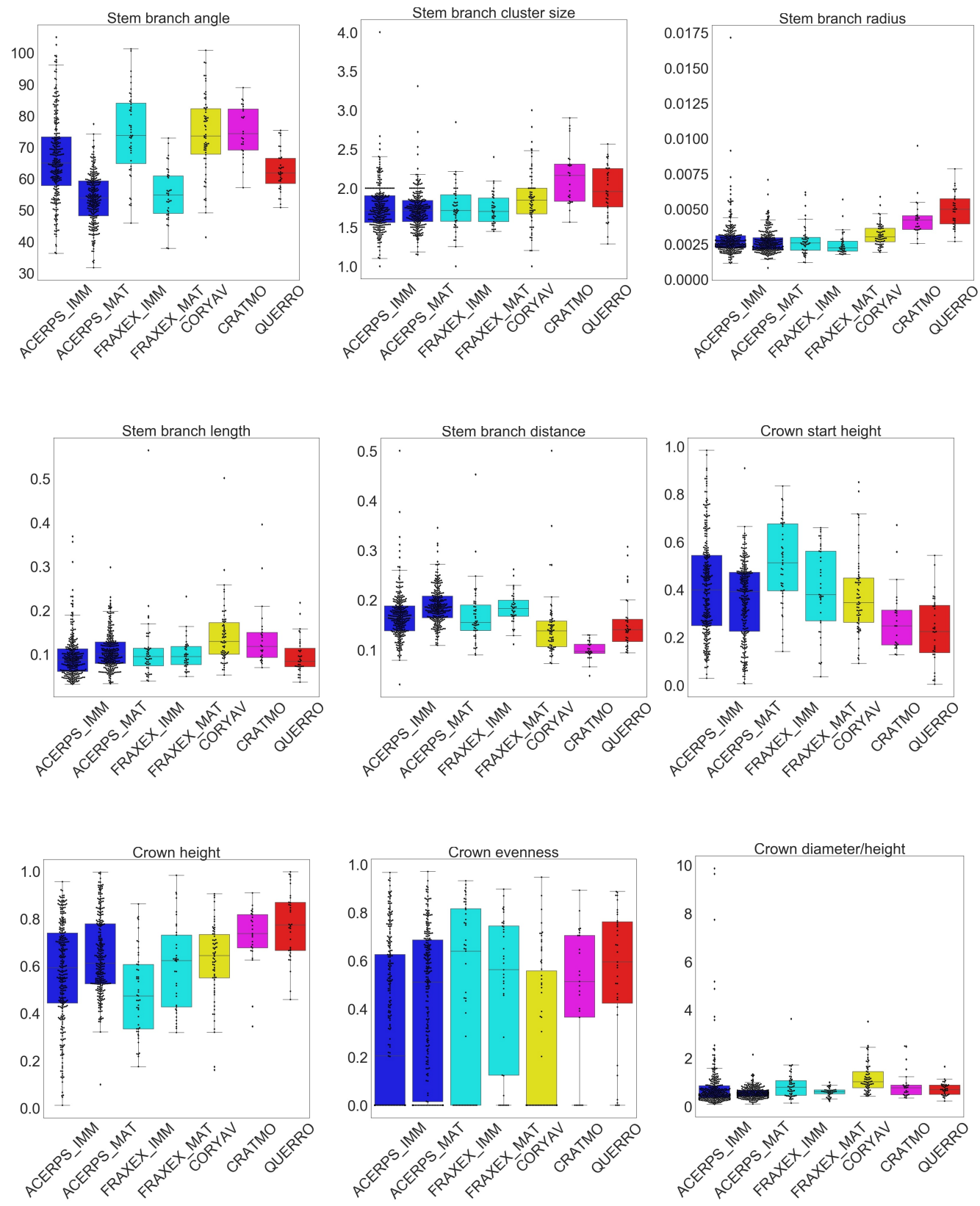

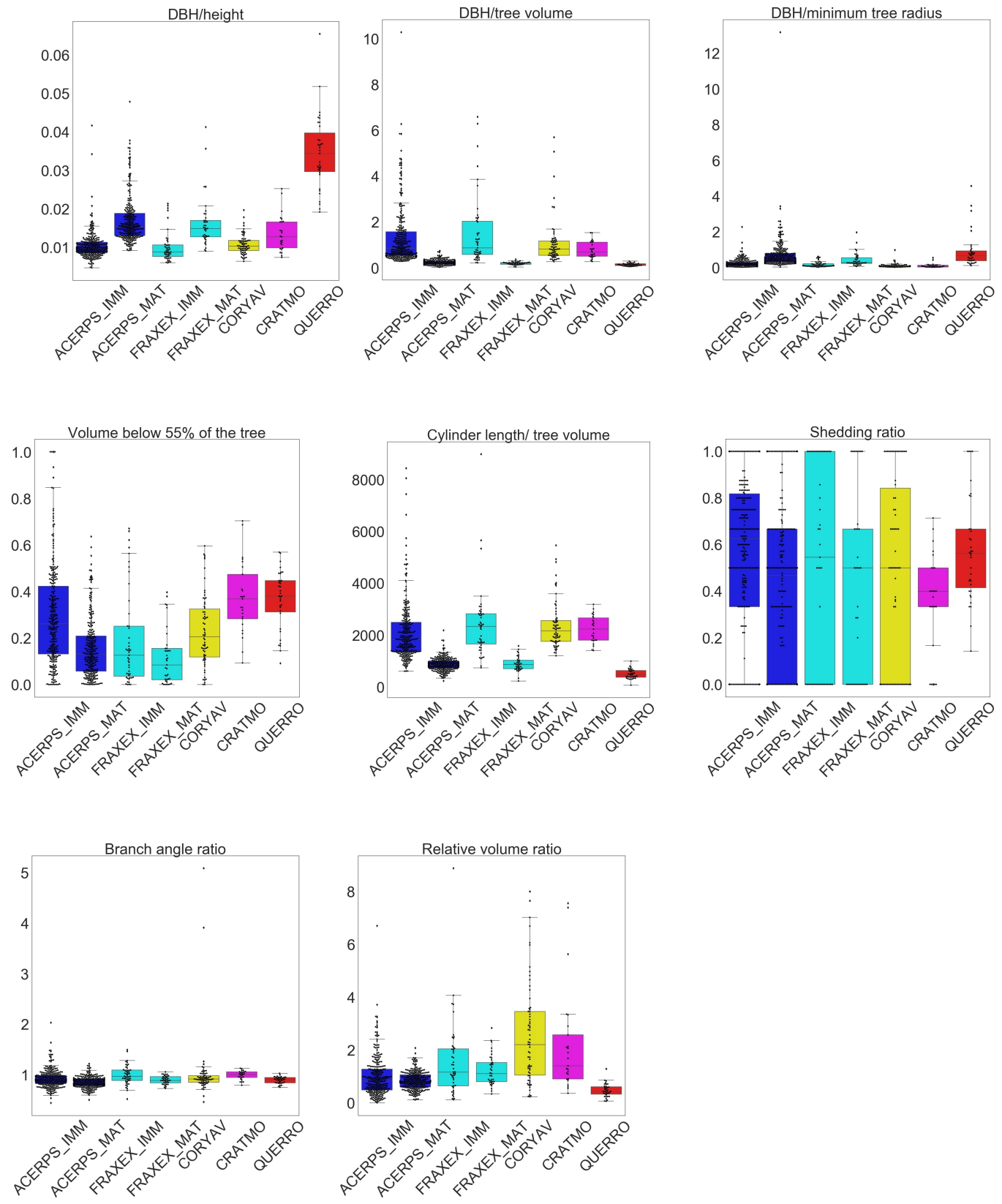

Figure 6: Boxplots and swarmplots of the different structural features for all the classes in scenario 2. From left to right in every boxplot: young ACERPS, mature ACERPS, young FRAXEX, mature FRAXEX, CORYAV, CRATMO, QUERRO. The vertical line inside the box is the median. Box limits give the 1st and 3rd quartiles of the distribution and the whiskers extend to 1.5 times the distance between the 1st and 3rd quartiles, or the distribution extremes 
For example, Fig. 7 shows that the stem branch angle (the median of the branching angles of the first order branches) is higher and more variable for small trees $(\mathrm{DBH}<0.2 \mathrm{~m})$ but it becomes lower and consistent for mature trees $(\mathrm{DBH}>0.2 \mathrm{~m})$. However, Lin and Herold (2016) and Åkerblom et al. (2017) explicitly mention normalising by the height or DBH to reduce the impact of tree age but did not mention the quality of the normalisation procedure nor any unexplained variance in the post-normalised data. This high variability for small trees and more constant values for larger trees was mainly observed for the tall tree species, ACERPS and FRAXEX, of which small and large trees were included in the dataset. MacFarlane and Kane (2017) also noticed that some architectural traits like stem slenderness and relative crown length and width appeared to be more variable among trees in light-limited functional groups i.e. young trees being overtopped by the large dominant trees of the same species. Ultimately, this size-dependency causes high intra-species variability, increasing classification error. 


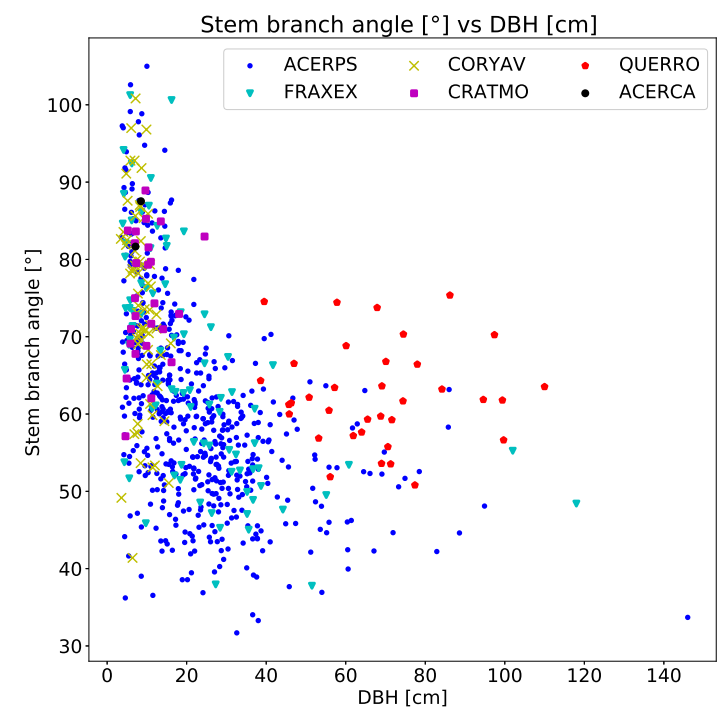

Figure 7: Scatterplot of Feature 1, the stem branch angle [ $\left.{ }^{\circ}\right]$, versus the DBH $[\mathrm{cm}]$ of the tree for the different tree species.

\subsection{Principal Component Analysis}

The first two important principal components explain $21.5 \%$ and $16.6 \%$ of the total variance within the dataset. Small trees in the understory, which include shrubs and young trees, have a higher PCA1 score than larger dominant trees (Fig. 8). There is also a high positive correlation of 0.80 between this axis and feature 14, the total branch length divided by the total branch volume. This feature relates to the branch slenderness which has been proven to be different for different canopy classes (understory, overtopped versus dominant) and thus different tree height (MacFarlane and Kane, 2017). MacFarlane and Kane (2017) note that the branch slenderness is a complex function of both species and canopy position and likely influences many aspects of tree 
function. However, PCA1 is also highly correlated to the crown start height (the branch free stem height), which resulted in slightly different values for QUERRO in comparison with ACERPS and FRAXEX, but is not obviously related to the canopy class. In contrast, the second axis (PCA2) could be related to shade-tolerance as it shows lower values for shade-tolerant species, while higher PCA2 values are obtained for more shade-intolerant trees.

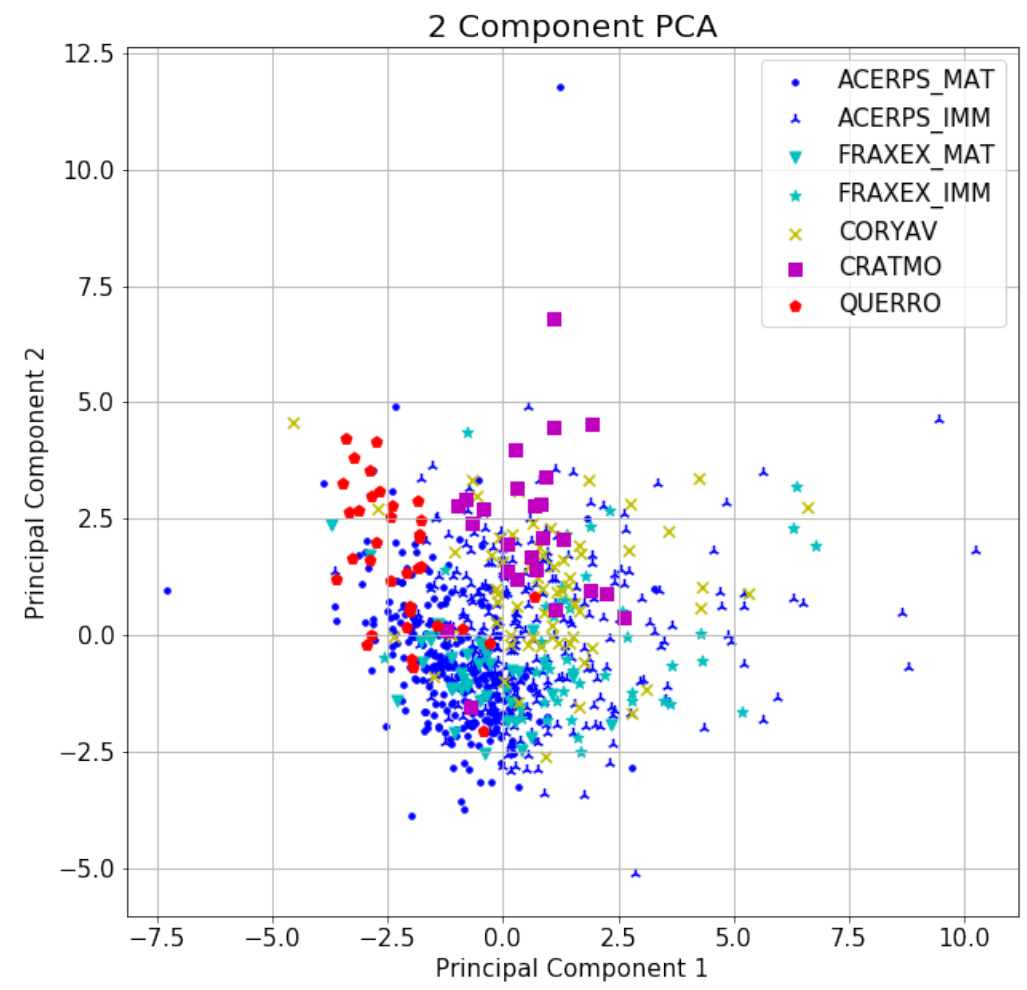

Figure 8: The scatterplot of the first two PCA-axes. Colour is according to species with small trees in a different symbol than large trees of ACERPS and FRAXEX. 


\subsection{Classification}

For every classification method in both scenario 1 (5 classes) and scenario 2 (7 classes) a mean test accuracy and aggregated confusion matrix were obtained. The mean test accuracies for scenario 1 (Table 4 ) did not vary much over the different classification methods, with the lowest score of $80 \%$ for the KNN-classifier and the highest score of $82 \%$ for the SVMrbf-classifier. T-tests confirm there are only significant differences between the mean test accuracy scores of the SVMrbf-classifier and those of the KNN-, SVMlinand SVMpoly-classifiers $(\alpha=0.01)$. The accuracies seem relatively high but are only $8.0 \%$ to $10 \%$ higher than the null accuracy $(72 \%)$ of assuming every tree being the dominant species. Producer accuracies reveal a different story (Fig. 9a). These show accuracies above 95\% for ACERPS, with little variation among the classifiers. The latter is most likely due to the large amount of observations obtained for ACERPS as misclassifying 10 ACERPS trees will not significantly affect the accuracy ratio. The QUERRO class is also relatively well classified but more variable, with accuracies between $69 \%$ and $91 \%$ depending on the classifier, as there were only 35 QUERRO observations. However, here we need to keep in mind that the $\mathrm{DBH}$ range of this class was limited to large, mature trees, thus simplifying the classification. The shrub species, CORYAV and CRATMO have similarly low accuracies between $40 \%$ and $60 \%$ except for the KNN-classifier which only classifies $20 \%$ of CRATMO trees correctly. These producer accuracies vary little despite the small amount of observations for these species. Very low producer accu- 
racies below $17 \%$ are obtained for FRAXEX which is mostly misclassified as ACERPS (Table 5). These producer accuries can be translated in high specificity values of more than $95 \%$ for all classifiers but low sensitivities ranging from $35 \%$ to $47 \%$. Similar results for scenario 2 show that splitting up the classes into age groups does not generally improve the mean test accuracy score (Table 4). There are no significant differences between the mean test accuracy scores of each method when comparing scenario 1 and $2(\alpha=0.01)$.

\begin{tabular}{|c|c|c|c|c|c|}
\hline \multicolumn{6}{|c|}{ Default class_weight } \\
\hline & \multicolumn{2}{|c|}{ Scenario 1 ( 5 classes $)$} & \multicolumn{2}{|c|}{ Scenario 2 ( 7 classes $)$} & Scenario 2 converted to Scenario 1 \\
\hline & Mean test accuracy (\%) & Standard deviation (pp) & Mean test accuracy (\%) & Standard deviation (pp) & Mean test accuracy (\%) \\
\hline KNN & 79.95 & 3.06 & 75.35 & 2.26 & 79.29 \\
\hline MLR & 80.87 & 2.95 & 79.04 & 2.15 & 80.87 \\
\hline SVMlin & 80.87 & 1.76 & 79.29 & 2.31 & 81.00 \\
\hline SVMrbf & 82.29 & 1.26 & 79.55 & 2.06 & 81.40 \\
\hline SVMpoly & 80.61 & 2.30 & 76.67 & 3.59 & 80.61 \\
\hline \multicolumn{6}{|c|}{ Balanced class_weight } \\
\hline MLR & 68.74 & 3.09 & 65.59 & 4.05 & 68.21 \\
\hline SVMlin & 74.03 & 3.61 & 69.89 & 4.27 & 72.16 \\
\hline SVMrbf & 78.50 & 1.05 & 75.60 & 2.78 & 77.44 \\
\hline SVMpoly & 78.88 & 1.61 & 72.58 & 2.08 & 76.52 \\
\hline
\end{tabular}

Table 4: Summarising table of the mean test accuracies (\%) and their standard deviations (percentage points) obtained for the different classification methods for both scenarios, in case of default and balanced class_weight. Also the mean test accuracies converted from the confusion matrices of scenario 2 to scenario 1 are shown.

Applying a balanced class weight increases the difference between different classifiers. T-tests confirm that only the mean test accuracy scores of SVMrbf and SVMpoly are not significantly different $(\alpha=0.01)$ when using balanced class weights. The results of the MLR-classifier and the SVMlinclassifier were affected most, since the mean test accuracies were about 12 and 6.9 percent lower for these classifiers respectively (Table 4). However, a balanced class weight also results in a shift in producer accuracies which was again largest in the MLR- and SVMlin-classifiers (Fig. 9). There is a 
trade-off between the accuracy for ACERPS (specificity) and the accuracies of the other species (sensitivity) (Fig. 9). The mean test accuracies might have diminished but a balanced class weight translates into more balanced producer accuracies compared to the default case. Moreover, when the balanced class weight is combined with scenario 2, the producer accuracies are even more balanced and all accuracies are above $56 \%$ and $58 \%$ for the MLR and SVMlin classifiers respectively (Fig. 9b).

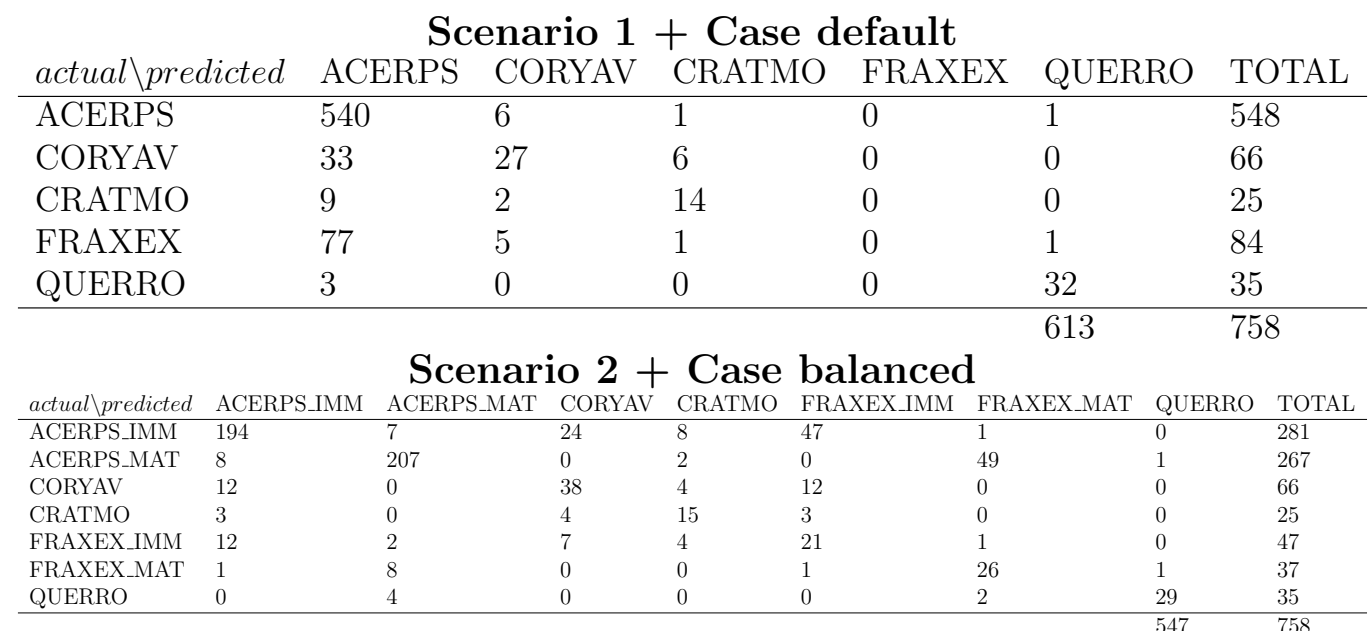

Table 5: Aggregated confusion matrix of the SVMlin-classifier in scenario 1 in case of default class_weight and in scenario 2 in the case of balanced class_weight. The rows are the actual species in the dataset while the columns are the predicted species. The total correctly classified number of trees is given in the bottom row on the left while the total number of trees is given in the bottom row on the right.

\section{Discussion}

Principal component analysis revealed that the largest effect on tree structure is related to canopy class and possibly shade-tolerance and not to species as such. However, we need to keep in mind the limited number of species 


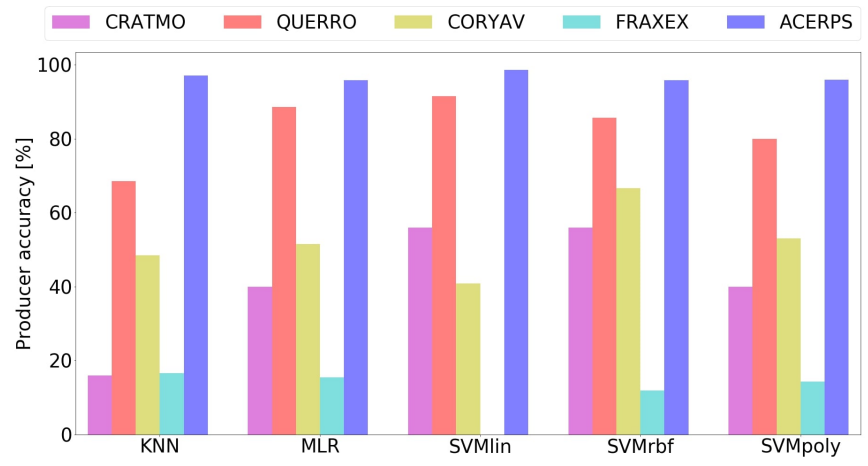

(a) Scenario $1+$ Case default

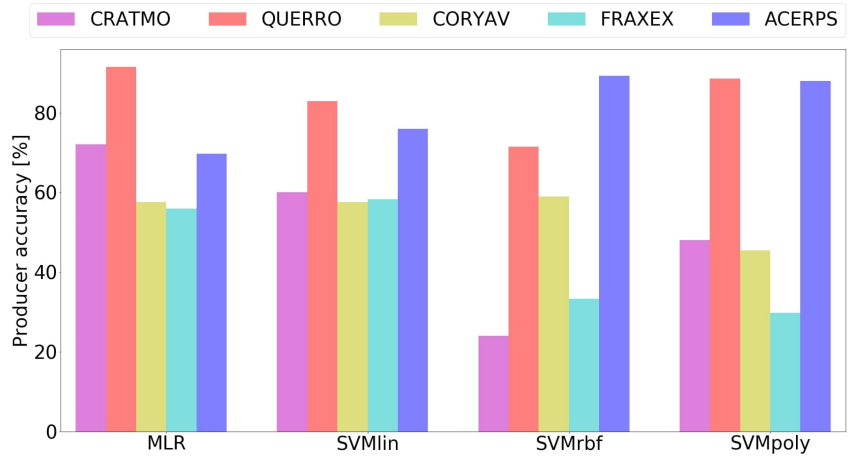

(b) Scenario $2+$ Case balanced

Figure 9: Producer accuracies for Scenario 1 (five classes) in the case of default class weight and for Scenario 2 (seven classes) in the case of balanced class weight. The species are represented in the order of increasing number of trees in the dataset

in the dataset. Nevertheless, this result makes sense as trees have to adapt to local ecological conditions. MacFarlane and Kane (2017) state that this adaptation leads to a convergence of tree traits across species. Ultimately the convergence results in lower inter-species variation, which complicates classification. These results support the findings of several studies that show that species that differ in their maximum height and light demand are found to differ in architectural traits (Poorter et al., 2006). Tall species grow effi- 
ciently in height to get access to sunlight and they do so by making a slender stem and a more narrow crown (Thomas, 1996; Kohyama et al., 2003; Poorter et al., 2003; Aiba and Nakashizuka, 2009). In contrast, small species enhance current light interception by making wide and long crowns (Kohyama et al., 2003; Poorter et al., 2003). Young trees of tall species, however, show more variability in their structural traits resembling traits of small species.

\subsection{Targeted application}

Crucial for the evaluation of a classification method is defining an ultimate objective. However, a specific objective or targeted application is often missing in classification studies (Fassnacht et al., 2016). In fact, the definition of optimum classification accuracy varies with the viewpoint of the user of the application. We might want to monitor the abundance of Acer pseudoplatanus (ACERPS) because this is a highly successful invader of semi-natural woodland in Wytham Woods (Butt et al., 2009). In this case a classification method resulting in a high producer accuracy for ACERPS (high specificity), such as a bimodal classifier, equals a successful result. All of the classification methods with a default class weight satisfy this demand. However, it should be kept in mind that the high specificity is combined with a low sensitivity resulting in an overestimation of ACERPS. On the other hand, we might want to monitor Fraxinus excelsior, which is a keystone tree species throughout temperate Europe but whose future existence is threatened by an emerging invasive fungal disease (Pautasso et al., 2013). Monitoring the abundance of 
FRAXEX requires high producer accuracies, and thus a high sensitivity, for this species. The presented features and classification methods do not fulfil this requirement as the maximum FRAXEX-accuracy was only $58 \%$ for the SVMlin-classifier for scenario 2 in the balanced case.

Most landscape level classification studies using airborne hyperspectral data, multi-spectral images or airborne LiDAR data cannot take into account understory tree species or very young trees. For example, Lee et al. (2016) mention focusing on mapping the six most dominant canopy tree species because subcanopy species and shrubs are hard to detect by airborne remote sensing methods. Therefore, the influence of these trees on classification has not been thoroughly investigated and the number of tree species classified has been limited. In contrast to the airborne remote sensing methods, TLS can include subcanopy trees and shrub species because it is a ground surface observation, although this viewpoint also results in a suboptimal characterisation of the tree crown. Nevertheless, surface measurement opens up new avenues for ecological studies and in turn a deeper understanding of the structure of trees enables more efficient classification. In fact, Fassnacht et al. (2016) recommend that future research efforts should focus more on this causal understanding of why tree species classification approaches work under certain conditions and why they do not in other cases. Our dataset and study are unique as it includes the classification of understory species and tries to obtain an understanding of the drivers of the tree structure. 


\subsection{Data fusion}

There was a loss of knowledge at several steps as, firstly, the QSMs are a summary of the point cloud data. The structural features, which are fed to the classification and PCA, are in their turn a summary of the information contained in the QSM. Nevertheless, the information contained in the 17 structural features is sufficient to obtain moderate classification results depending on the targeted application. But, these structural features are clearly not sufficient to separate Acer pseudoplatanus and Fraxinus excelsior, which are structurally very similar trees. This could be the result of the choice or formulation of these features, which may not be the best ones for describing all the information, causing the overlap between different species. The normalisation, which was mainly done by dividing by the DBH or height, could also be in some cases better and more natural when dividing by another variable, such as the DBH or height squared. Therefore, other structural measures should be studied in order to further explore the classification from TLS data alone. However, the convergence in tree and branch architecture along a gradient of crowding and shading for individuals of broad-leaved species as observed by MacFarlane and Kane (2017) makes species classification, based on solely structural features, difficult.

To achieve good classification results for every application, the combination of these structural features with other data sources should be considered. Hyperspectral data has, for example, shown potential for tree species clas- 
sification and shown to be complementary to LiDAR data (Dalponte et al., 2008; Jones et al., 2010; Alonzo et al., 2014). Fusion of airborne and groundbased data is, however, still a technical challenge and has not been fully investigated for TLS. Although our classification method is fully automatic, collecting TLS data to build the QSMs is time consuming and has only been done for limited areas of forest. Future development of mobile laser scanning systems can potentially speed up the data collection process. Moreover, Fassnacht et al. (2016) showed that there are few examples of tree species classification over a large geographical extent. They also state that bridging the gap between current approaches and tree species inventories over these extents still remains one of the biggest challenges of this research field. Under the explicit assumption that field plots represent their surrounding environment, field inventory plots have been used consistently to extrapolate the understanding of the forest structure at plot level to landscape-to-regional scales. The use of high-resolution remote sensing is a useful additional data source for extrapolating field plot forest dynamics to larger scales. Fusion of unmanned aerial vehicle (UAV) and terrestrial LiDAR data can potentially be used for the 3D mapping of forest structure at plot to landscape levels, which will be key for the reduction of uncertainties in forest structure and carbon estimates (Brede et al., 2019).

Only a few classification studies, such as Korpela et al. (2014), systematically describe and examine the traits that drive the observed variance in 
the remote sensing signal and thereby enable or hamper species classification (Fassnacht et al., 2016). Our main focus was on the variance introduced by the tree-size. This size effect has not been thoroughly investigated in classification before, as only canopy trees were considered previously due to sensor limitations. However, size clearly affects the structural features that were used to classify the tree species. But, the size of the tree is not the only factor which influences the structure of trees. In the future other influencing factors, such as the presence of neighbouring trees, competition, wind, soil conditions, snow etc. have to be taken into account (Holbrook and Putz, 1989; Mäkelä and Vanninen, 1998; MacFarlane and Kane, 2017).

\subsection{Limitations and prospects}

This study considered every tree of the five main tree species, including all sizes and shrub species, of a 1.4 ha plot in a mixed deciduous forest which has been managed in a specific way. Therefore, the results might not be applicable to more or less intensely managed woodlands even of the same species composition. In this study the computed features were not specifically validated. For the most basic ones, such as the $\mathrm{DBH}$ and height of the tree, there have been many studies validating automated TLS derivation (Calders et al., 2014, 2015b). Other features should still be validated, especially the features that concern the top part of the tree, which are well known to be more occluded in TLS measurements. However, for the purpose 
of species classification the key concern is that computed values can separate the species and not that the values are correct in absolute terms. Nevertheless, the classification of species that can only be separated by the top of their crown need accurate crown characterisation. In this respect, we should try to find crown features that are robust and less sensitive to poor TLS data quality. The combination of drone LiDAR could help here if the TLS and drone point clouds could be successfully combined.

More species from different forest types should be considered for future studies. Large tropical trees are particularly challenging to reconstruct from TLS data due to the complexity of trunk shapes (e.g. buttresses) and to higher occlusion levels in tree crowns (Takoudjou et al., 2018). The classification of species in an evergreen forest could also prove difficult due to the presence of leaves which decreases the quality of the QSMs. In addition, as the structure can be influenced by several factors, such as the nutrient content of the soil and climatic factors, future work should test the methodology in different sites and plots to gain a better view on its applicability.

\section{Conclusion}

We examined the accuracy of tree species classification, in a mixed deciduous forest plot including understory species, based on structural features extracted from TLS-derived QSMs. The analysis revealed two factors complicating classification. On the one hand, size-dependency increases intra- 
species variability while on the other hand the convergence of structural tree traits across species of the same canopy class and shade tolerance group decreases inter-species variability. Mean test accuracies around 80\% were achieved while producer accuracies ranged from 0 to $60 \%$ for three of the five species. A trade-off between sensitivity and specificity, was made to improve these producer accuracies obtaining more than $57 \%$ for all species. However, determining the classification performance and best classification method greatly depends on the application. These results bring us one step closer to the integration of automated species identification from TLS into forest inventories and thereby contributing to the effective management of forested ecosystems. The additional information on the specific form of a tree, presented by the structural features, also gives more insight into the ecological interactions between trees and their environment. In this way TLS data opens up potential for testing and further development of ecological theory.

\section{Acknowledgments}

The collection of the Wytham Woods TLS data was funded through the Metrology for Earth Observation and Climate project (MetEOC-2), grant number ENV55 within the European Metrology Research Programme (EMRP). The EMRP is jointly funded by the EMRP participating countries within EURAMET and the European Union. Mathias Disney acknowledges capital funding from NERC NCEO and UCL for TLS equipment and from NERC standard grants NE/N00373X/1 and NE/P011780/1. Pasi Raumonen ac- 
knowledges funding from 3DForMod project, ERA-NET FACCE ERA-GAS.

\section{Data availability}

The Wytham Woods 3D models are online available from https://bitbucket . org/tree_research/wytham_woods_3d_model/.

\section{References}

Aiba, M., Nakashizuka, T., 2009. Architectural differences associated with adult stature and wood density in 30 temperate tree species. Functional Ecology 23, 265-273.

Åkerblom, M., Raumonen, P., Mäkipää, R., Kaasalainen, M., 2017. Automatic tree species recognition with quantitative structure models. Remote Sensing of Environment 191, 1-12. URL: http://www. sciencedirect. com/science/article/pii/S0034425716304746, doi:https://doi.org/ 10.1016/j.rse.2016.12.002.

Alonzo, M., Bookhagen, B., Roberts, D.A., 2014. Urban tree species mapping using hyperspectral and lidar data fusion. Remote Sensing of Environment 148, $70-83$. URL: http://www.sciencedirect. com/science/article/pii/S0034425714001047, doi:https://doi.org/ $10.1016 / j . r s e .2014 .03 .018$.

Bonan, G.B., 2008. Forests and climate change: Forcings, feedbacks, and the climate benefits of forests. Science 320, 1444- 
1449. URL: http://science.sciencemag.org/content/320/5882/ 1444, doi:10.1126/science.1155121.

Brede, B., Calders, K., Lau, A., Raumonen, P., Bartholomeus, H.M., Herold, M., Kooistra, L., 2019. Non-destructive tree volume estimation through quantitative structure modelling: Comparing uav laser scanning with terrestrial lidar. Remote Sensing of Environment 233, 111355. URL: http://www. sciencedirect.com/science/article/ pii/S0034425719303748, doi:https://doi.org/10.1016/j.rse.2019. 111355.

Burt, A., Disney, M., Calders, K., 2019. Extracting individual trees from lidar point clouds using treeseg. Methods in Ecology and Evolution 10, 438-445.

Butt, N., Campbell, G., Malhi, Y., Morecroft, M., Fenn, K., Thomas, M., 2009. Initial results from establishment of a long-term broadleaf monitoring plot at wytham woods, oxford, uk. University Oxford, Oxford, UK .

Calders, K., Armston, J., Newnham, G., Herold, M., Goodwin, N., 2014. Implications of sensor configuration and topography on vertical plant profiles derived from terrestrial lidar. Agricultural and Forest Meteorology 194, 104 - 117. URL: http://www.sciencedirect.com/science/article/ pii/S0168192314000902, doi:https://doi.org/10.1016/j.agrformet. 2014.03 .022 . 
Calders, K., Disney, M., Nightingale, J., Origo, N., Barker, A., Raumonen, P., Lewis, P., Burt, A., Brennan, J., Fox, N., 2015a. Traceability of essential climate variables through forest stand reconstruction with terrestrial laser scanning, in: Proceedings of SilviLaser 2015, pp. 122-124.

Calders, K., Newnham, G., Burt, A., Murphy, S., Raumonen, P., Herold, M., Culvenor, D., Avitabile, V., Disney, M., Armston, J., Kaasalainen, M., 2015b. Nondestructive estimates of above-ground biomass using terrestrial laser scanning. Methods in Ecology and Evolution 6, 198208. URL: https://besjournals.onlinelibrary.wiley.com/doi/abs/ 10.1111/2041-210X.12301, doi:10.1111/2041-210X.12301.

Calders, K., Origo, N., Burt, A., Disney, M., Nightingale, J., Raumonen, P., Åkerblom, M., Malhi, Y., Lewis, P., 2018. Realistic forest stand reconstruction from terrestrial lidar for radiative transfer modelling. Remote Sensing 10. URL: http://www.mdpi.com/2072-4292/10/6/933, doi:10.3390/rs10060933.

Dalponte, M., Bruzzone, L., Gianelle, D., 2008. Fusion of hyperspectral and lidar remote sensing data for classification of complex forest areas. IEEE Transactions on Geoscience and Remote Sensing 46, 1416-1427. doi:10. 1109/TGRS. 2008.916480.

Deshayes, M., Guyon, D., Jeanjean, H., Stach, N., Jolly, A., Hagolle, O., 2006. The contribution of remote sensing to the assessment of drought 
effects in forest ecosystems. Ann. For. Sci. 63, 579-595. URL: https: //doi.org/10.1051/forest:2006045, doi:10.1051/forest:2006045.

Duriqi, R., Raca, V., Cico, B., 2016. Comparative analysis of classification algorithms on three different datasets using weka, in: 2016 5th Mediterranean Conference on Embedded Computing (MECO), IEEE. pp. 335-338.

Fassnacht, F.E., Latifi, H., Stereńczak, K., Modzelewska, A., Lefsky, M., Waser, L.T., Straub, C., Ghosh, A., 2016. Review of studies on tree species classification from remotely sensed data. Remote Sensing of Environment 186, $64-87$. URL: http://www. sciencedirect. com/science/article/pii/S0034425716303169, doi:https://doi.org/ $10.1016 / j . r s e .2016 .08 .013$.

Fender, A.C., Gansert, D., Jungkunst, H.F., Fiedler, S., Beyer, F., Schützenmeister, K., Thiele, B., Valtanen, K., Polle, A., Leuschner, C., 2013. Root-induced tree species effects on the source/sink strength for greenhouse gases (ch4, n2o and co2) of a temperate deciduous forest soil. Soil Biology and Biochemistry 57, 587 597. URL: http://www.sciencedirect.com/science/article/pii/ S0038071712003069, doi:https://doi.org/10.1016/j.soilbio. 2012 . 08.004 .

Forest GEO Global Earth Observatory Network, 2019. Wytham Woods ForestGEO. https://forestgeo.si.edu/sites/europe/wytham-woods. Online; accessed 18 Novemberl 2019. 
Gong, P., Pu, R., Yu, B., 1997. Conifer species recognition: An exploratory analysis of in situ hyperspectral data. Remote Sensing of Environment 62, 189 - 200. URL: http://www.sciencedirect. com/science/article/pii/S0034425797000941, doi:https://doi.org/ 10.1016/S0034-4257(97)00094-1.

Hobbie, S.E., Reich, P.B., Oleksyn, J., Ogdahl, M., Zytkowiak, R., Hale, C., Karolewski, P., 2006. Tree species effects on decomposition and forest floor dynamics in a common garden. Ecology 87, 2288-2297. URL: https://esajournals.onlinelibrary.wiley.com/doi/abs/10.1890/ 0012-9658\\%282006\\%2987\\%5B2288\\%3ATSEODA \\%5D2. 0. CO \\%3B2, doi:10 . 1890/0012-9658(2006)87 [2288: TSEODA] 2. 0 . CO ; 2 .

Holbrook, N.M., Putz, F.E., 1989. Influence of neighbors on tree form: Effects of lateral shade and prevention of sway on the allometry of liquidambar styraciflua (sweet gum). American Journal of Botany 76, 17401749. URL: https://bsapubs . onlinelibrary.wiley.com/doi/abs/10. 1002/j.1537-2197.1989.tb15164.x, doi:10.1002/j.1537-2197.1989. tb15164.x.

James, G., Witten, D., Hastie, T., Tibshirani, R., 2013. An introduction to statistical learning. volume 112. Springer.

Jones, T.G., Coops, N.C., Sharma, T., 2010. Assessing the utility of airborne hyperspectral and lidar data for species distribution mapping in the coastal pacific northwest, canada. Remote Sensing of 
Environment 114, 2841 - 2852. URL: http://www.sciencedirect. com/science/article/pii/S003442571000218X, doi:https://doi.org/ $10.1016 / j . r s e .2010 .07 .002$.

Keenan, R.J., 2015. Climate change impacts and adaptation in forest management: a review. Annals of Forest Science 72, 145167. URL: https://doi.org/10.1007/s13595-014-0446-5, doi:10. 1007/s13595-014-0446-5.

Kohyama, T., Suzuki, E., Partomihardjo, T., Yamada, T., Kubo, T., 2003. Tree species differentiation in growth, recruitment and allometry in relation to maximum height in a bornean mixed dipterocarp forest. Journal of Ecology 91, 797-806. doi:10.1046/j.1365-2745.2003.00810.x.

Korpela, I., Mehtatalo, L., Markelin, L., Seppanen, A., Kangas, A., 2014. Tree species identification in aerial image data using directional reflectance signatures. Silva Fennica 48. doi:10.14214/sf .1087.

Lee, J., Cai, X., Lellmann, J., Dalponte, M., Malhi, Y., Butt, N., Morecroft, M., Schönlieb, C., Coomes, D.A., 2016. Individual tree species classification from airborne multisensor imagery using robust pca. IEEE Journal of Selected Topics in Applied Earth Observations and Remote Sensing 9, 2554-2567. doi:10.1109/JSTARS . 2016. 2569408.

Liao, Y., Vemuri, V., 2002. Use of k-nearest neighbor classifier for intrusion detection11an earlier version of this paper is to appear in the proceedings 
of the 11th usenix security symposium, san francisco, ca, august 2002. Computers \& Security 21, 439 - 448. URL: http://www. sciencedirect. com/science/article/pii/S016740480200514X, doi:https://doi.org/ 10.1016/S0167-4048(02)00514-X.

Lin, Y., Herold, M., 2016. Tree species classification based on explicit tree structure feature parameters derived from static terrestrial laser scanning data. Agricultural and Forest Meteorology 216, 105 - 114. URL: http://www.sciencedirect.com/science/article/ pii/S016819231500742X, doi:https://doi.org/10.1016/j.agrformet. 2015.10 .008 .

MacFarlane, D.W., Kane, B., 2017. Neighbour effects on tree architecture: functional trade-offs balancing crown competitiveness with wind resistance. Functional Ecology 31, 1624-1636. URL: https://besjournals.onlinelibrary.wiley.com/doi/abs/10. 1111/1365-2435.12865, doi:10.1111/1365-2435.12865.

Mäkelä, A., Vanninen, P., 1998. Impacts of size and competition on tree form and distribution of aboveground biomass in scots pine. Canadian Journal of Forest Research 28, 216-227.

Newby, D., Freitas, A.A., Ghafourian, T., 2013. Coping with unbalanced class data sets in oral absorption models. Journal of chemical information and modeling 53, 461-474. 
Newnham, G.J., Armston, J.D., Calders, K., Disney, M.I., Lovell, J.L., Schaaf, C.B., Strahler, A.H., Danson, F.M., 2015. Terrestrial laser scanning for plot-scale forest measurement. Current Forestry Reports 1, 239-251. URL: https://doi.org/10.1007/s40725-015-0025-5, doi:10. $1007 / \mathrm{s} 40725-015-0025-5$.

Othmani, A., Voon, L.F.L.Y., Stolz, C., Piboule, A., 2013. Single tree species classification from terrestrial laser scanning data for forest inventory. Pattern Recognition Letters 34, 2144 - 2150. URL: http:// WWW.sciencedirect.com/science/article/pii/S0167865513002997, doi:https ://doi .org/10.1016/j.patrec.2013.08.004.

Pautasso, M., Aas, G., Queloz, V., Holdenrieder, O., 2013. European ash (fraxinus excelsior) dieback - a conservation biology challenge. Biological Conservation 158, 37 - 49. URL: http://www.sciencedirect. com/science/article/pii/S0006320712003813, doi:https://doi.org/ $10.1016 /$ j.biocon .2012 .08 .026 .

Pedregosa, F., Varoquaux, G., Gramfort, A., Michel, V., Thirion, B., Grisel, O., Blondel, M., Prettenhofer, P., Weiss, R., Dubourg, V., et al., 2011. Scikit-learn: Machine learning in python. Journal of machine learning research $12,2825-2830$.

Poorter, L., Bongers, F., Sterck, F.J., Wöll, H., 2003. Architecture of 53 rain forest tree species differing in adult stature and shade tolerance. Ecology 
84, 602-608. doi:10.1890/0012-9658(2003)084 [0602: AORFTS] 2.0.CO;

2.

Poorter, L., Bongers, L., Bongers, F., 2006. Architecture of 54-moist-forest tree species: traits, trade-offs, and functional groups. Ecology 87, 12891301. doi:10.1890/0012-9658(2006)87 [1289:AOMTST] 2.0.CO;2.

Pozzolo, A.D., Caelen, O., Johnson, R.A., Bontempi, G., 2015. Calibrating probability with undersampling for unbalanced classification, in: 2015 IEEE Symposium Series on Computational Intelligence, pp. 159-166. doi:10.1109/SSCI.2015.33.

Puttonen, E., Suomalainen, J., Hakala, T., Räikkönen, E., Kaartinen, H., Kaasalainen, S., Litkey, P., 2010. Tree species classification from fused active hyperspectral reflectance and lidar measurements. Forest Ecology and Management 260, 1843 - 1852. URL: http://www.sciencedirect. com/science/article/pii/S0378112710004901, doi:https://doi.org/ $10.1016 / j$. foreco. 2010.08 .031 .

Raumonen, P., 2019. Inversetampere/treeqsm: Version 2.3.1. https://doi. org/10.5281/zenodo.3482908. doi:10.5281/zenodo. 3482908 .

Raumonen, P., Kaasalainen, M., Åkerblom, M., Kaasalainen, S., Kaartinen, H., Vastaranta, M., Holopainen, M., Disney, M., Lewis, P., 2013. Fast automatic precision tree models from terrestrial laser scanner data. Remote 
Sensing 5, 491-520. URL: http://www.mdpi.com/2072-4292/5/2/491, doi:10.3390/rs5020491.

Story, M., Congalton, R.G., 1986. Accuracy assessment: a user's perspective. Photogrammetric Engineering and remote sensing 52, 397-399.

Takoudjou, S.M., Ploton, P., Sonké, B., Hackenberg, J., Griffon, S., De Coligny, F., Kamdem, N.G., Libalah, M., Mofack, G.I., Le Moguédec, G., et al., 2018. Using terrestrial laser scanning data to estimate large tropical trees biomass and calibrate allometric models: A comparison with traditional destructive approach. Methods in Ecology and Evolution 9, 905-916.

The ECN Data Centre, 2019. Site Information: Wytham. http://data.ecn . ac.uk/sites/ecnsites. asp?site=T08. Online; accessed 1 April 2019.

Thomas, S.C., 1996. Reproductive allometry in malaysian rain forest trees: Biomechanics versus optimal allocation. Evolutionary Ecology 10, 517-530. URL: https://doi.org/10.1007/BF01237882, doi:10.1007/ $\mathrm{BF} 01237882$.

Van Rossum, G., Drake Jr, F.L., 1995. Python tutorial. Centrum voor Wiskunde en Informatica Amsterdam, The Netherlands.

Varma, S., Simon, R., 2006. Bias in error estimation when using crossvalidation for model selection. BMC bioinformatics 7, 91 . 
Vesterdal, L., Schmidt, I.K., Callesen, I., Nilsson, L.O., Gundersen, P., 2008. Carbon and nitrogen in forest floor and mineral soil under six common european tree species. Forest Ecology and Management 255, 35 - 48. URL: http://www.sciencedirect.com/ science/article/pii/S037811270700610X, doi:https://doi.org/10. 1016/j.foreco.2007.08.015. 